Article

\title{
On Some New Properties of the Fundamental Solution to the Multi-Dimensional Space- and Time-Fractional Diffusion-Wave Equation
}

\author{
Yuri Luchko ${ }^{\dagger}$ \\ Beuth University of Applied Sciences Berlin, 13353 Berlin, Germany; luchko@beuth-hochschule.de; \\ Tel.: +49-30-4504-5295 \\ + Current address: Beuth Hochschule für Technik Berlin, Fachbereich II Mathematik-Physik-Chemie, \\ Luxemburger Str. 10, 13353 Berlin, Germany.
}

Received: 11 November 2017; Accepted: 4 December 2017; Published: 8 December 2017

\begin{abstract}
In this paper, some new properties of the fundamental solution to the multi-dimensional space- and time-fractional diffusion-wave equation are deduced. We start with the Mellin-Barnes representation of the fundamental solution that was derived in the previous publications of the author. The Mellin-Barnes integral is used to obtain two new representations of the fundamental solution in the form of the Mellin convolution of the special functions of the Wright type. Moreover, some new closed-form formulas for particular cases of the fundamental solution are derived. In particular, we solve the open problem of the representation of the fundamental solution to the two-dimensional neutral-fractional diffusion-wave equation in terms of the known special functions.
\end{abstract}

Keywords: multi-dimensional diffusion-wave equation; neutral-fractional diffusion-wave equation; fundamental solution; Mellin-Barnes integral; integral representation; Wright function; generalized Wright function

MSC: 26A33; 35C05; 35E05; 35L05; 45K05; 60E99

\section{Introduction}

Partial fractional differential equations are nowadays both an important research subject and a popular modeling approach. Despite of importance of mathematical models in two- and three-dimensions for applications, most of the recent publications devoted to fractional diffusion-wave equations have dealt with the one-dimensional case. The literature dealing with multi-dimensional partial fractional differential equations is still not numerous and can be divided into several groups, those devoted to Cauchy problems on the whole space, boundary-value problems on bounded domains, and of course to different types of equations, including single- and multi-term equations as well as equations of distributed order. Because the focus of this paper is on the Cauchy problem for a model linear time- and space-fractional diffusion-wave equation, we mention here only some important relevant publications.

The fundamental solution to the multi-dimensional time-fractional diffusion-wave equation with the Laplace operator was derived for the first time by Kochubei in [1] and Schneider and Wyss in [2] independently from each other in terms of the Fox H-function. We note that in [1], the Cauchy problem for the general fractional diffusion equation with the regularized fractional derivative (the Caputo fractional derivative in modern terminology) and the general second-order spatial differential operator was also investigated. In the series of publications [3-5], Hanyga considered mathematical, physical, and probabilistic properties of the fundamental solutions to multi-dimensional time-, space- and space-time-fractional diffusion-wave equations, respectively. Recently, Luchko and his co-authors 
started to employ the method of the Mellin-Barnes integral representation to derive further properties of the multi-dimensional space-time-fractional diffusion-wave equation (see, e.g., [6-10]). Still, the list of the properties, particular cases, integral and series representations, asymptotic formulas, and so forth known for the fundamental solution to the one-dimensional diffusion-wave equation (see, e.g., [11]) is essentially more expanded compared to the multi-dimensional case, and thus further investigations of the multi-dimensional case are required.

In this paper, some new properties and particular cases of the fundamental solution to the multi-dimensional space- and time-fractional diffusion-wave equation are deduced. In the second section, we recall the Mellin-Barnes representations of the fundamental solution that were derived in the previous publications of the author and his co-authors. In the third section, the Mellin-Barnes integral is used to obtain two new representations of the fundamental solution in the form of the Mellin convolution of the special functions of the Wright type. The fourth section is devoted to the derivation of some new closed-form formulas for the fundamental solution. In particular, the open problem of the representation of the fundamental solution to the two-dimensional neutral-fractional diffusion-wave equation in terms of the known elementary or special functions is solved.

\section{Problem Formulation and Auxiliary Results}

In this section, we present a problem formulation and some auxiliary results that are used in the rest of the paper.

\subsection{Problem Formulation}

In this paper, we deal with the multi-dimensional space- and time-fractional diffusion-wave equation in the following form:

$$
D_{t}^{\beta} u(\mathrm{x}, t)=-(-\Delta)^{\frac{\alpha}{2}} u(\mathrm{x}, t), \quad \mathrm{x} \in \mathbb{R}^{n}, t>0,1<\alpha \leq 2,0<\beta \leq 2,
$$

where $(-\Delta)^{\frac{\alpha}{2}}$ is the fractional Laplacian and $D_{t}^{\beta}$ is the Caputo time-fractional derivative of order $\beta$.

The Caputo time-fractional derivative of order $\beta>0$ is defined by the formula

$$
D_{t}^{\beta} u(x, t)=\left(I_{t}^{n-\beta} \frac{\partial^{n} u}{\partial t^{n}}\right)(t), \quad n-1<\beta \leq n, n \in \mathbb{N},
$$

where $I_{t}^{\gamma}$ is the Riemann-Liouville fractional integral:

$$
\left(I_{t}^{\gamma} u\right)(t)=\left\{\begin{array}{l}
\frac{1}{\Gamma(\gamma)} \int_{0}^{t}(t-\tau)^{\gamma-1} u(\mathrm{x}, \tau) d \tau \text { for } \gamma>0 \\
u(\mathrm{x}, t) \text { for } \gamma=0
\end{array}\right.
$$

The fractional Laplacian $(-\Delta)^{\frac{\alpha}{2}}$ is defined as a pseudo-differential operator with the symbol $|\kappa|^{\alpha}([12,13])$ :

$$
\left(\mathcal{F}(-\Delta)^{\frac{\alpha}{2}} f\right)(\kappa)=|\kappa|^{\alpha}(\mathcal{F} f)(\kappa),
$$

where $(\mathcal{F} f)(\kappa)$ is the Fourier transform of a function $f$ at the point $\kappa \in \mathbb{R}^{n}$ defined by

$$
(\mathcal{F} f)(\kappa)=\hat{f}(\kappa)=\int_{\mathbb{R}^{n}} e^{i \kappa \cdot x} f(\mathrm{x}) d \mathrm{x} .
$$

For $0<\alpha<m, m \in \mathbb{N}$ and $x \in \mathbb{R}^{n}$, the fractional Laplacian can be also represented as a hypersingular integral ([13]):

$$
(-\Delta)^{\frac{\alpha}{2}} f(\mathrm{x})=\frac{1}{d_{n, m}(\alpha)} \int_{\mathbb{R}^{n}} \frac{\left(\Delta_{\mathrm{h}}^{m} f\right)(\mathrm{x})}{|\mathrm{h}|^{n+\alpha}} d \mathrm{~h}
$$


with a suitably defined finite-differences operator $\left(\Delta_{\mathrm{h}}^{m} f\right)(\mathrm{x})$ and a normalization constant $d_{n, m}(\alpha)$.

According to [13], the representation given by Equation (5) of the fractional Laplacian in the form of the hypersingular integral does not depend on $m, m \in \mathbb{N}$ provided that $\alpha<m$.

We note that in the one-dimensional case, Equation (1) is a particular case of a more general equation with the Caputo time-fractional derivative and the Riesz-Feller space-fractional derivative that was discussed in detail in [11]. For $\alpha=2$, the fractional Laplacian $(-\Delta)^{\frac{\alpha}{2}}$ is simply $-\Delta$, and thus Equation (1) is a particular case of the time-fractional diffusion-wave equation that was considered in many publications, including, for example, [1,2,5,14-17]. For $\alpha=2$ and $\beta=1$, Equation (1) is reduced to the diffusion equation, and for $\alpha=2$ and $\beta=2$, it is the wave equation that justifies its denotation as a fractional diffusion-wave equation.

In this paper, we deal with the Cauchy problem for Equation (1) with Dirichlet initial conditions. If the order $\beta$ of the time-derivative satisfies the condition $0<\beta \leq 1$, we pose an initial condition of the form

$$
u(\mathrm{x}, 0)=\varphi(\mathrm{x}), \quad \mathrm{x} \in \mathbb{R}^{n} .
$$

For the orders $\beta$ satisfying the condition $1<\beta \leq 2$, the second initial condition in the form

$$
\frac{\partial u}{\partial t}(\mathrm{x}, 0)=0, \quad \mathrm{x} \in \mathbb{R}^{n}
$$

is added to the Cauchy problem.

Because the initial-value problem given by Equations (1) and (6) (or (1), (6) and (7)) is linear, its solution can be represented in the form

$$
u(\mathrm{x}, t)=\int_{\mathbb{R}^{n}} G_{\alpha, \beta, n}(\mathrm{x}-\zeta, t) \varphi(\zeta) d \zeta,
$$

where $G_{\alpha, \beta, n}$ is the first fundamental solution to the fractional diffusion-wave Equation (1), that is, the solution to the problem given by Equations (1) and (6) with the initial condition

$$
u(\mathrm{x}, 0)=\prod_{i=1}^{n} \delta\left(x_{i}\right), \quad \mathrm{x}=\left(x_{1}, x_{2}, \ldots, x_{n}\right) \in \mathbb{R}^{n}
$$

or to the problem given by Equations (1), (6) and (7) with the initial conditions

$$
u(\mathrm{x}, 0)=\prod_{i=1}^{n} \delta\left(x_{i}\right), \quad \mathrm{x}=\left(x_{1}, x_{2}, \ldots, x_{n}\right) \in \mathbb{R}^{n}
$$

and

$$
\frac{\partial u}{\partial t}(\mathrm{x}, 0)=0, \quad \mathrm{x} \in \mathbb{R}^{n}
$$

for $0<\beta \leq 1$ or $1<\beta \leq 2$, respectively, with $\delta$ being the Dirac delta function.

Thus the behavior of the solutions to the problem given by Equations (1) and (6) (or (1), (6) and (7)) is determined by the fundamental solution $G_{\alpha, \beta, n}(x, t)$, and the focus of this paper is on the derivation of the new properties of the fundamental solution.

\subsection{Mellin-Barnes Representations of the Fundamental Solution}

A Mellin-Barnes representation of the fundamental solution to the multi-dimensional space- and time-fractional diffusion-wave Equation (1) was derived for the first time in [7] for the case $\beta=\alpha$ (see also [8]), in [10] for the case $\beta=\alpha / 2$, and in [9] for the general case. For the reader's convenience, we present here a short schema of its derivation.

The application of the multi-dimensional Fourier transform (4) with respect to the spatial variable $\mathrm{x} \in \mathbb{R}^{n}$ to Equation (1) and to the initial conditions given by Equation (6) with $\varphi(\mathrm{x})=\prod_{i=1}^{n} \delta\left(x_{i}\right)$, and 
Equation (7) (the last condition is relevant only if $\beta>1$ ) leads to the ordinary fractional differential equation in the Fourier domain:

$$
D_{t}^{\beta} \hat{G}_{\alpha, \beta, n}(\kappa, t)+|\kappa|^{\alpha} \hat{G}_{\alpha, \beta, n}(\kappa, t)=0,
$$

along with the initial conditions

$$
\hat{G}_{\alpha, \beta, n}(\kappa, 0)=1
$$

in the case for $0<\beta \leq 1$, or with the initial conditions

$$
\hat{G}_{\alpha, \beta, n}(\kappa, 0)=1, \frac{\partial}{\partial t} \hat{G}_{\alpha, \beta, n}(\kappa, 0)=0
$$

in the case for $1<\beta \leq 2$.

In both cases, the unique solution of Equation (8) with the initial conditions given by Equations (9) or (9) and (10) has the following form (see, e.g., [18]):

$$
\hat{G}_{\alpha, \beta, n}(\kappa, t)=E_{\beta}\left(-|\kappa|^{\alpha} t^{\beta}\right)
$$

in terms of the Mittag-Leffler function $E_{\beta}(z)$ that is defined by a convergent series:

$$
E_{\beta}(z)=\sum_{n=0}^{\infty} \frac{z^{n}}{\Gamma(1+\beta n)}, \quad \beta>0, z \in \mathbb{C}
$$

Because of the asymptotic formula (see, e.g., [19]):

$$
E_{\beta}(-x)=-\sum_{k=1}^{m} \frac{(-x)^{-k}}{\Gamma(1-\beta k)}+O\left(|x|^{-1-m}\right), m \in \mathbb{N}, x \rightarrow+\infty, 0<\beta<2
$$

we have the inclusion $\hat{G}_{\alpha, \beta, n} \in L_{1}\left(\mathbb{R}^{n}\right)$ under the condition $\alpha>1$, and thus the inverse Fourier transform of Equation (11) can be represented as follows:

$$
G_{\alpha, \beta, n}(\mathrm{x}, t)=\frac{1}{(2 \pi)^{n}} \int_{\mathbb{R}^{n}} e^{-i \kappa \cdot \mathrm{x}} E_{\beta}\left(-|\kappa|^{\alpha} t^{\beta}\right) d \kappa, \quad \mathrm{x} \in \mathbb{R}^{n}, t>0 .
$$

Because $E_{\beta}\left(-|\kappa|^{\alpha} t^{\beta}\right)$ is a radial function, the known formula (see, e.g., [13])

$$
\frac{1}{(2 \pi)^{n}} \int_{\mathbb{R}^{n}} e^{-i \kappa \cdot \mathrm{x}} \varphi(|\kappa|) d \kappa=\frac{|\mathbf{x}|^{1-\frac{n}{2}}}{(2 \pi)^{\frac{n}{2}}} \int_{0}^{\infty} \varphi(\tau) \tau^{\frac{n}{2}} J_{\frac{n}{2}-1}(\tau|\mathbf{x}|) d \tau
$$

for the Fourier transform of the radial functions can be applied, where $J_{v}$ denotes the Bessel function with index $v$ (for the properties of the the Bessel function, see, e.g., [20]), and we arrive at the representation

$$
G_{\alpha, \beta, n}(x, t)=\frac{|x|^{1-\frac{n}{2}}}{(2 \pi)^{\frac{n}{2}}} \int_{0}^{\infty} E_{\beta}\left(-\tau^{\alpha} t^{\beta}\right) \tau^{\frac{n}{2}} J_{\frac{n}{2}-1}(\tau|x|) d \tau
$$

whenever the integral in Equation (16) converges absolutely or at least conditionally.

The representation given by Equation (16) can be transformed to a Mellin-Barnes integral.

We start with the case $|x|=0(x=(0, \ldots, 0))$ and obtain the formula

$$
G_{\alpha, \beta, n}(0, t)=\frac{1}{(2 \pi)^{n}} \int_{\mathbb{R}^{n}} E_{\beta}\left(-|\kappa|^{\alpha} t^{\beta}\right) d \kappa
$$


which can be represented in the form

$$
G_{\alpha, \beta, n}(0, t)=\frac{1}{(2 \pi)^{n}} \frac{2 \pi^{\frac{n}{2}}}{\Gamma\left(\frac{n}{2}\right)} \int_{0}^{\infty} E_{\beta}\left(-\tau^{\alpha} t^{\beta}\right) \tau^{n-1} d \tau
$$

as a result of the known formula (see, e.g., [13]):

$$
\int_{\mathbb{R}^{n}} f(|\mathbf{x}|) d \mathbf{x}=\frac{2 \pi^{\frac{n}{2}}}{\Gamma\left(\frac{n}{2}\right)} \int_{0}^{\infty} \tau^{n-1} f(\tau) d \tau
$$

The asymptotics of the Mittag-Leffler function ensures convergence of the integral in Equation (17) under the condition $0<n<\alpha$. Thus, for $1<\alpha \leq 2$, the fundamental solution $G_{\alpha, \beta, n}$ is finite at $|x|=0$ only in the one-dimensional case and we obtain the formula

$$
G_{\alpha, \beta, 1}(0, t)=\frac{t^{-\frac{\beta}{\alpha}}}{\alpha \pi} \int_{0}^{\infty} E_{\beta}(-u) u^{\frac{1}{\alpha}-1} d u=\frac{t^{-\frac{\beta}{\alpha}} \Gamma\left(\frac{1}{\alpha}\right) \Gamma\left(1-\frac{1}{\alpha}\right)}{\Gamma \pi},
$$

which is valid for $\alpha>1$ if $0<\beta<2$ and for $\alpha>2$ if $\beta=2$. This formula is an easy consequence from the known Mellin integral transform of the Mittag-Leffler function (see, e.g., [21,22]):

$$
\int_{0}^{\infty} E_{\beta}(-u) u^{s-1} d u=\frac{\Gamma(s) \Gamma(1-s)}{\Gamma(1-\beta s)} \text { if }\left\{\begin{array}{l}
0<\Re(s)<1 \text { for } 0<\beta<2 \\
0<\Re(s)<1 / 2 \text { for } \beta=2 .
\end{array}\right.
$$

The Mellin integral transform plays an important role in fractional calculus in general and for the derivation of the results of this paper in particular; thus we recall the definitions of the Mellin transform and the inverse Mellin transform, respectively:

$$
\begin{gathered}
f^{*}(s)=(\mathcal{M} f(\tau))(s)=\int_{0}^{\infty} f(\tau) \tau^{s-1} d \tau, \quad \tau>0, \\
f(\tau)=\left(\mathcal{M}^{-1} f^{*}(s)\right)(\tau)=\frac{1}{2 \pi i} \int_{\gamma-i \infty}^{\gamma+i \infty} f^{*}(s) \tau^{-s} d s, \gamma_{1}<\Re(s)=\gamma<\gamma_{2} .
\end{gathered}
$$

The Mellin integral transform exists in particular for the functions continuous on the intervals $(0, \varepsilon]$ and $[E,+\infty)$ and integrable on the interval $(\epsilon, E)$ with any $\varepsilon, E, 0<\varepsilon<E<+\infty$ that satisfy the estimates $|f(\tau)| \leq M_{1} \tau^{-\gamma_{1}}$ for $0<\tau<\varepsilon$ and $|f(\tau)| \leq M_{2} \tau^{-\gamma_{2}}$ for $\tau>E$ with $\gamma_{1}<\gamma_{2}$ and some constants $M_{1}$ and $M_{2}$. In this case, the Mellin integral transform $f^{*}(s)$ is analytic in the vertical strip $\gamma_{1}<\Re(s)=\gamma<\gamma_{2}$.

If $f$ is piecewise differentiable and $\tau^{\gamma-1} f(\tau) \in L^{c}(0, \infty)$, then Equation (21) holds at all points of continuity for $f$. The integral in Equation (21) has to be considered in the sense of the Cauchy principal value.

For the general theory of the Mellin integral transform, we refer the reader to [22]. Several applications of the Mellin integral transform in fractional calculus are discussed in $[7,21]$.

If the dimension $n$ of Equation (1) is greater that one, the fundamental solution $G_{\alpha, \beta, n}(x, t)$ has an integrable singularity at the point $|x|=0$.

Now we proceed with the case $x \neq 0$ and first discuss the convergence of the integral in the integral representation given by Equation (16). It follows from the asymptotic formulas for the Mittag-Leffler function and the known asymptotic behavior of the Bessel function (see, e.g., [20]) that the integral in Equation (16) converges conditionally in the case $n<2 \alpha+1$ and absolutely in the case $n<2 \alpha-1$. Thus for $1<\alpha \leq 2$ and $n=1,2,3$, the integral in Equation (16) is at least conditionally convergent. 
Now the technique of the Mellin integral transform is applied to deduce a Mellin-Barnes representation of the fundamental solution $G_{\alpha, \beta, n}(\mathrm{x}, t)$. In particular, we use the convolution theorem for the Mellin integral transform that reads as

$$
\int_{0}^{\infty} f_{1}(\tau) f_{2}\left(\frac{y}{\tau}\right) \frac{d \tau}{\tau} \stackrel{\mathcal{M}}{\longleftrightarrow} f_{1}^{*}(s) f_{2}^{*}(s),
$$

where by $\stackrel{\mathcal{M}}{\longleftrightarrow}$ the juxtaposition of a function $f$ with its Mellin transform $f^{*}$ is denoted.

It can be easily seen that for $x \neq 0$ the integral on the right-hand side of Equation (16) is the Mellin convolution of the functions

$$
f_{1}(\tau)=E_{\beta}\left(-\tau^{\alpha} t^{\beta}\right) \quad \text { and } \quad f_{2}(\tau)=\frac{|x|^{-n}}{(2 \pi)^{\frac{n}{2}}} \tau^{-\frac{n}{2}-1} J_{\frac{n}{2}-1}\left(\frac{1}{\tau}\right)
$$

at the point $y=\frac{1}{|x|}$.

The Mellin transform of the Mittag-Leffler function (Equation (19)), the known Mellin integral transform of the Bessel function ([22]):

$$
J_{v}(2 \sqrt{\tau}) \stackrel{\mathcal{M}}{\longleftrightarrow} \frac{\Gamma(v / 2+s)}{\Gamma(v / 2+1-s)},-\Re(v / 2)<\Re(s)<3 / 4,
$$

and some elementary properties of the Mellin integral transform (see, e.g., [21,22]) lead to the Mellin transform formulas:

$$
\begin{array}{r}
f_{1}^{*}(s)=\frac{t^{-\frac{\beta}{\alpha} s}}{\alpha} \frac{\Gamma\left(\frac{s}{\alpha}\right) \Gamma\left(1-\frac{s}{\alpha}\right)}{\Gamma\left(1-\frac{\beta}{\alpha} s\right)}, \quad 0<\Re(s)<\alpha, \\
f_{2}^{*}(s)=\frac{|\mathbf{x}|^{-n}}{(2 \pi)^{\frac{n}{2}}}\left(\frac{1}{2}\right)^{-\frac{n}{2}+s} \frac{\Gamma\left(\frac{n}{2}-\frac{s}{2}\right)}{\Gamma\left(\frac{s}{2}\right)}, \quad \frac{n}{2}-\frac{1}{2}<\Re(s)<n .
\end{array}
$$

These two formulas, the convolution Theorem (Equation (22)) for the Mellin transform, and the inverse Mellin transform Equation (21) result in the following Mellin-Barnes integral representation of the fundamental solution $G_{\alpha, \beta, n}$ :

$$
G_{\alpha, \beta, n}(x, t)=\frac{1}{\alpha} \frac{|x|^{-n}}{\pi^{\frac{n}{2}}} \frac{1}{2 \pi i} \int_{\gamma-i \infty}^{\gamma+i \infty} \frac{\Gamma\left(\frac{n}{2}-\frac{s}{2}\right) \Gamma\left(\frac{s}{\alpha}\right) \Gamma\left(1-\frac{s}{\alpha}\right)}{\Gamma\left(1-\frac{\beta}{\alpha} s\right) \Gamma\left(\frac{s}{2}\right)}\left(\frac{2 t^{\frac{\beta}{\alpha}}}{|x|}\right)^{-s} d s,
$$

where $\frac{n}{2}-\frac{1}{2}<\gamma<\min (\alpha, n)$. We note that the Mellin-Barnes integral given by Equation (23) can also be interpreted as a particular case of the Fox H-function. The theory of the H-function, its properties, and applications are presented in a number of textbooks and papers (see, e.g., [23-28]); thus, here we do not discuss this subject in detail and prefer to directly deduce the properties of the fundamental solution $G_{\alpha, \beta, n}$ from its Mellin-Barnes representation (Equation (23)). Starting with this representation and using simple linear variables' substitutions, we can easily derive some other forms of this representation that will be useful for further discussions. For example, the substitutions $s \rightarrow-s$ and then $s \rightarrow s-n$ in the Mellin-Barnes representation given by Equation (23) result in two other equivalent representations:

$$
G_{\alpha, \beta, n}(\mathrm{x}, t)=\frac{1}{\alpha} \frac{|\mathrm{x}|^{-n}}{\pi^{\frac{n}{2}}} \frac{1}{2 \pi i} \int_{\gamma-i \infty}^{\gamma+i \infty} \frac{\Gamma\left(\frac{n}{2}+\frac{s}{2}\right) \Gamma\left(-\frac{s}{\alpha}\right) \Gamma\left(1+\frac{s}{\alpha}\right)}{\Gamma\left(1+\frac{\beta}{\alpha} s\right) \Gamma\left(-\frac{s}{2}\right)}\left(\frac{|\mathrm{x}|}{2 t^{\frac{\beta}{\alpha}}}\right)^{-s} d s
$$


and

$$
G_{\alpha, \beta, n}(\mathrm{x}, t)=\frac{1}{\alpha} \frac{t^{-\frac{\beta n}{\alpha}}}{(4 \pi)^{\frac{n}{2}}} \frac{1}{2 \pi i} \int_{\gamma-i \infty}^{\gamma+i \infty} \frac{\Gamma\left(\frac{s}{2}\right) \Gamma\left(\frac{n}{\alpha}-\frac{s}{\alpha}\right) \Gamma\left(1-\frac{n}{\alpha}+\frac{s}{\alpha}\right)}{\Gamma\left(1-\frac{\beta}{\alpha} n+\frac{\beta}{\alpha} s\right) \Gamma\left(\frac{n}{2}-\frac{s}{2}\right)}\left(\frac{|\mathrm{x}|}{2 t^{\frac{\beta}{\alpha}}}\right)^{-s} d s
$$

under the conditions $-\min (\alpha, n)<\gamma<\frac{1}{2}-\frac{n}{2}$ or $\max (n-\alpha, 0)<\gamma<n$, respectively.

Finally, we demonstrate how these integral representations can be used, for example, for deriving some series representations of $G_{\alpha, \beta, n}(\mathrm{x}, t)$ and then its representations in terms of elementary or special functions of the hypergeometric type. To this end, we consider a simple example. In the case $\beta=1$ and $\alpha=2$ (standard diffusion equation), the representation given by Equation (25) takes the following form (two pairs of the gamma functions in the integral on the right-hand side of Equation (25) are canceled):

$$
G_{2,1, n}(x, t)=\frac{t^{-\frac{n}{2}}}{2(4 \pi)^{\frac{n}{2}}} \frac{1}{2 \pi i} \int_{\gamma-i \infty}^{\gamma+i \infty} \Gamma\left(\frac{s}{2}\right)\left(\frac{z}{2}\right)^{-s} d s, z=\frac{|x|}{\sqrt{t}} .
$$

The substitution of the variables $s \rightarrow 2 s$ leads to an even simpler representation:

$$
G_{2,1, n}(\mathrm{x}, t)=\frac{t^{-\frac{n}{2}}}{(4 \pi)^{\frac{n}{2}}} \frac{1}{2 \pi i} \int_{\gamma-i \infty}^{\gamma+i \infty} \Gamma(s)\left(\frac{z}{2}\right)^{-2 s} d s, z=\frac{|x|}{\sqrt{t}} .
$$

According to the Cauchy theorem, the contour of integration in the integral on the right-hand side of the previous formula can be transformed to the loop $L_{-\infty}$ starting and ending at $-\infty$ and encircling all poles $s_{k}=-k, k=0,1,2, \ldots$ of the function $\Gamma(s)$. Taking into account the Jordan lemma, the formula

$$
\operatorname{res}_{s=-k} \Gamma(s)=\frac{(-1)^{k}}{k !}, k=0,1,2, \ldots
$$

and the Cauchy residue theorem lead to a series representation of $G_{2,1, n}(x, t)$ :

$$
G_{2,1, n}(x, t)=\frac{t^{-\frac{n}{2}}}{(4 \pi)^{\frac{n}{2}}} \int_{\gamma-i \infty}^{\gamma+i \infty} \Gamma(s)\left(\frac{z}{2}\right)^{-2 s} d s=\frac{t^{-\frac{n}{2}}}{(4 \pi)^{\frac{n}{2}}} \sum_{k=0}^{\infty} \frac{(-1)^{k}}{k !}\left(\frac{z}{2}\right)^{2 k}, z=\frac{|x|}{\sqrt{t}} .
$$

Thus the fundamental solution $G_{2,1, n}$ to the $n$-dimensional diffusion equation takes its standard form:

$$
G_{2,1, n}(\mathrm{x}, t)=\frac{1}{(\sqrt{4 \pi t})^{n}} \exp \left(-\frac{|\mathrm{x}|^{2}}{4 t}\right) .
$$

\subsection{Special Functions of the Wright Type}

The fundamental solutions to different time-, space-, or time- and space-fractional partial differential equations are closely connected to the special functions of hypergeometric type. In the general situation, some particular cases of the Fox H-function are often involved (see, e.g., [1,2]). However, for particular cases of the orders of the fractional derivatives, the H-function can sometimes be reduced to some simpler special functions, mainly of Wright type (see, e.g., [29] for the one-dimensional case of the time-fractional diffusion-wave equation). Because the Fox $\mathrm{H}$-function has still not been investigated in all its details and, in particular, because no packages for its numerical calculation are available, this reduction is very welcome. In this paper, some new reduction formulas for the fundamental solution to the multi-dimensional time- and space-fractional diffusion-wave Equation (1) are derived. In this subsection, we shortly discuss the special functions of the Wright type that appear in these derivations. For more details regarding theory and applications of these special functions, we refer the reader to, for example, [30-36].

We start with the Wright function:

$$
W_{a, \mu}(z)=\sum_{k=0}^{\infty} \frac{z^{k}}{k ! \Gamma(a+\mu k)}, \mu>-1, a, z \in \mathbb{C}
$$


that was introduced for the first time in [37] for the case $\mu>0$. In particular, in [37,38], Wright investigated some elementary properties and asymptotic behavior of this function in connection with his research on the asymptotic theory of partitions.

Because of the relation

$$
J_{v}(z)=\left(\frac{z}{2}\right)^{v} W_{1+v, 1}\left(-\frac{1}{4} z^{2}\right)
$$

the Wright function can be considered as a generalization of the Bessel function $J_{v}(z)$. In turn, the Wright function is a particular case of the Fox H-function (see, e.g., [25,39]):

$$
W_{a, \mu}(-z)=H_{0,2}^{1,0}\left[z \mid \begin{array}{c}
- \\
(0,1),(1-a, \mu)
\end{array}\right] .
$$

The Wright function is an entire function for all real values of the parameter $\mu$ (both positive and negative) under the condition $-1<\mu$, but its asymptotic behavior is different in the cases $\mu>0$, $\mu=0$, and $\mu<0$ (see [40] for details).

Two particular cases of the Wright function, namely, the functions $M(z ; \beta)=W_{1-\beta,-\beta}(-z)$ and $F(z ; \beta)=W_{0,-\beta}(-z)$ with the parameter $\beta$ between 0 and 1 , have been introduced and investigated in detail in $[41,42]$. These functions play an important role as fundamental solutions of the Cauchy and signaling problems for the one-dimensional time-fractional diffusion-wave equation ([29]).

In this paper, a four-parameter Wright function in the form

$$
W_{(a, \mu),(b, v)}(z):=\sum_{k=0}^{\infty} \frac{z^{k}}{\Gamma(a+\mu k) \Gamma(b+v k)}, \mu, v \in \mathbb{R}, a, b, z \in \mathbb{C}
$$

is also used. Wright himself investigated this function in [43] for the case $\mu>0, v>0$. For $a=\mu=1$ or $b=v=1$, the four-parameter Wright function is reduced to the Wright function (Equation (27)). In [44], Luchko and Gorenflo investigated the four-parameter Wright function for the first time in the case for which one of the parameters $\mu$ or $v$ is negative. In particular, they proved that the function $W_{(a, \mu),(b, v)}(z)$ is an entire function provided that $0<\mu+v, a, b \in \mathbb{C}$.

It is important to emphasize that the function $W_{(a, u),(b, v)}(z)$ can have an algebraic asymptotic expansion on the positive real semi-axis in the case of suitably restricted parameters (see [44] for details):

$$
\begin{gathered}
W_{(a, \mu),(b, v)}(x)=\sum_{l=0}^{L-1} \frac{x^{(a-1-l) /(-\mu)}}{(-\mu) \Gamma(l+1) \Gamma(b+v(a-l-1) /(-\mu))} \\
-\sum_{k=1}^{P} \frac{x^{-k}}{\Gamma(b-v k) \Gamma(a-\mu k)}+\mathrm{O}\left(x^{(a-1-L) /(-\mu)}\right)+\mathrm{O}\left(x^{-1-P}\right), \quad x \rightarrow+\infty
\end{gathered}
$$

when $0<v / 3<-\mu<v \leq 2, L, P \in \mathbb{N}$.

In the important case $\mu+v=0$, the four-parameter Wright function is no longer an entire function. Indeed, in this case, the convergence radius of the series from Equation (30) is equal to 1 rather than to infinity, as can be seen from the asymptotics of the series terms as $k \rightarrow \infty$ :

$$
\begin{gathered}
\left|\frac{1}{\Gamma(a-v k) \Gamma(b+v k)}\right|=\left|\frac{\sin (\pi(a-v k))}{\pi} \frac{\Gamma(1-a+v k)}{\Gamma(b+v k)}\right|= \\
\left|\frac{\cosh (\pi \Im(a))}{\pi}(v k)^{1-a-b}\left[1+O\left(k^{-1}\right)\right]\right|, k \rightarrow+\infty .
\end{gathered}
$$

In the chain of the equalities above, the following known formulas for the gamma function were employed:

$$
\frac{\Gamma(z)}{\Gamma(1-z)}=\frac{\pi}{\sin (\pi z)}
$$




$$
\frac{\Gamma(s+a)}{\Gamma(s+b)}=s^{a-b}\left[1+O\left(s^{-1}\right)\right],|s| \rightarrow+\infty,|\arg (s)|<\pi
$$

Finally, we mention here the generalized Wright function that is defined by the following series (in the case of its convergence):

$$
{ }_{p} \Psi_{q}\left[\begin{array}{c}
\left(a_{1}, A_{1}\right), \ldots,\left(a_{p}, A_{p}\right) \\
\left(b_{1}, B_{1}\right) \ldots\left(b_{q}, B_{q}\right)
\end{array} ; z\right]=\sum_{k=0}^{\infty} \frac{\prod_{i=1}^{p} \Gamma\left(a_{i}+A_{i} k\right)}{\prod_{i=1}^{q} \Gamma\left(b_{i}+B_{i} k\right)} \frac{z^{k}}{k !}
$$

This function was introduced and investigated by Wright in [43]. For details regarding the generalized Wright function, we refer the readers to the recent book [45].

\section{New Integral Representations of the Fundamental Solution}

In the previous section, we derived the following integral representation of the fundamental solution:

$$
G_{\alpha, \beta, n}(\mathrm{x}, t)=\frac{|\mathrm{x}|^{1-\frac{n}{2}}}{(2 \pi)^{\frac{n}{2}}} \int_{0}^{\infty} E_{\beta}\left(-\tau^{\alpha} t^{\beta}\right) \tau^{\frac{n}{2}} J_{\frac{n}{2}-1}(\tau|\mathrm{x}|) d \tau .
$$

In this section, we demonstrate how the Mellin-Barnes representations of the fundamental solution can be employed to obtain other integral representations of the same type. The idea is very simple. For example, we start with the Mellin-Barnes representation given by Equation (25) and consider the kernel function:

$$
L_{\alpha, \beta, n}(s)=\frac{\Gamma\left(\frac{s}{2}\right) \Gamma\left(\frac{n}{\alpha}-\frac{s}{\alpha}\right) \Gamma\left(1-\frac{n}{\alpha}+\frac{s}{\alpha}\right)}{\Gamma\left(1-\frac{\beta}{\alpha} n+\frac{\beta}{\alpha} s\right) \Gamma\left(\frac{n}{2}-\frac{s}{2}\right)} .
$$

When the kernel function is represented as a product of two factors, the convolution theorem for the Mellin integral transform can be applied, and we obtain an integral representation of $G_{\alpha, \beta, n}$ of the type given by Equation (33). For example, we obtained the integral representation given by Equation (33) by employing the Mellin integral transform formulas for the Mittag-Leffler function and for the Bessel function, that is, by representing the kernel function $L_{\alpha, \beta, n}(s)$ as the following product:

$$
L_{\alpha, \beta, n}(s)=\frac{\Gamma\left(\frac{n}{\alpha}-\frac{s}{\alpha}\right) \Gamma\left(1-\frac{n}{\alpha}+\frac{s}{\alpha}\right)}{\Gamma\left(1-\frac{\beta}{\alpha} n+\frac{\beta}{\alpha} s\right)} \times \frac{\Gamma\left(\frac{s}{2}\right)}{\Gamma\left(\frac{n}{2}-\frac{s}{2}\right)} .
$$

In what follows, we consider other possibilities of the representation of the kernel function $L_{\alpha, \beta, n}(s)$ as a product of two factors. Of course, these factors should be chosen in a way that makes it possible to easily obtain the inverse Mellin integral transform of these factors in terms of the known elementary or special functions. In the following theorem, two possible representations are given.

Theorem 1. Let the inequalities $1<\alpha \leq 2,0<\beta \leq 2$ hold true. Then the first fundamental solution $G_{\alpha, \beta, n}$ of the multi-dimensional space-and time-fractional diffusion-wave Equation (1) has the following integral representations of the Mellin convolution type:

$$
\begin{gathered}
G_{\alpha, \beta, n}(\mathrm{x}, t)=\frac{1}{(\sqrt{\pi}|\mathrm{x}|)^{n}} \int_{0}^{\infty} e^{-\tau} \tau^{\frac{n}{2}-1} W_{(1, \beta),(0,-\alpha / 2)}\left(-\frac{\tau^{\alpha / 2} t^{\beta}}{(|\mathrm{x}| / 2)^{\alpha}}\right) d \tau \text { if } \beta>\alpha / 2, \\
G_{\alpha, \beta, n}(\mathrm{x}, t)=\frac{1}{(\sqrt{\pi}|\mathrm{x}|)^{n}} \int_{0}^{\infty} W_{\frac{\alpha}{2}, \frac{\alpha}{2}}(-\tau)_{1} \Psi_{1}\left[\begin{array}{c}
\left(\frac{n}{2}, \frac{\alpha}{2}\right) \\
(1, \beta)
\end{array} ; \frac{\tau t^{\beta}}{(|\mathrm{x}| / 2)^{\alpha}}\right] d \tau .
\end{gathered}
$$


Proof. To make calculations easier, we first perform the variables' substitution $s \rightarrow 2 s$ in the integral representation given by Equation (25). We obtain

$$
G_{\alpha, \beta, n}(\mathrm{x}, t)=\frac{2}{\alpha} \frac{t^{-\frac{\beta n}{\alpha}}}{(4 \pi)^{\frac{n}{2}}} \frac{1}{2 \pi i} \int_{\gamma-i \infty}^{\gamma+i \infty} \frac{\Gamma(s) \Gamma\left(\frac{n}{\alpha}-\frac{2}{\alpha} s\right) \Gamma\left(1-\frac{n}{\alpha}+\frac{2}{\alpha} s\right)}{\Gamma\left(1-\frac{\beta}{\alpha} n+\frac{2 \beta}{\alpha} s\right) \Gamma\left(\frac{n}{2}-s\right)}\left(z^{2}\right)^{-s} d s, z=\frac{|x|}{2 t^{\frac{\beta}{\alpha}}} .
$$

Now we represent the kernel function of the previous integral as follows:

$$
L_{\alpha, \beta, n}(s)=\Gamma(s) \times \frac{\Gamma\left(\frac{n}{\alpha}-\frac{2}{\alpha} s\right) \Gamma\left(1-\frac{n}{\alpha}+\frac{2}{\alpha} s\right)}{\Gamma\left(1-\frac{\beta}{\alpha} n+\frac{2 \beta}{\alpha} s\right) \Gamma\left(\frac{n}{2}-s\right)} .
$$

The inverse Mellin integral transform of $\Gamma(s)$ is simply the exponential function $\exp (-\tau)$ ([22]):

$$
f_{1}(\tau)=\frac{1}{2 \pi i} \int_{\gamma-i \infty}^{\gamma+i \infty} \Gamma(s) \tau^{-s} d s=e^{-\tau}
$$

To calculate the inverse Mellin transform of the second factor, the variables' substitution $s \rightarrow \frac{\alpha}{2} s$ is first applied. We then obtain the formula

$$
f_{2}(\tau)=\frac{\alpha}{2} \frac{1}{2 \pi i} \int_{\gamma-i \infty}^{\gamma+i \infty} \frac{\Gamma\left(\frac{n}{\alpha}-s\right) \Gamma\left(1-\frac{n}{\alpha}+s\right)}{\Gamma\left(1-\frac{\beta}{\alpha} n+\beta s\right) \Gamma\left(\frac{n}{2}-\frac{\alpha}{2} s\right)}\left(\tau^{\frac{\alpha}{2}}\right)^{-s} d s .
$$

To obtain a series representation of the function $f_{2}$, we employ the standard technique for the Mellin-Barnes integrals. According to the Cauchy theorem, the contour of integration in the integral on the right-hand side of the previous formula can be transformed to the loop $L_{+\infty}$ starting and ending at $+\infty$ and encircling all poles $s_{k}=k+\frac{n}{\alpha}, k=0,1,2, \ldots$ of the function $\Gamma\left(\frac{n}{\alpha}-s\right)$. Taking into account the Jordan lemma and the formula for the residual of the gamma function, the Cauchy residue theorem leads to a series representation of $f_{2}$ :

$$
f_{2}(\tau)=\frac{\alpha}{2} \sum_{k=0}^{\infty} \frac{(-1)^{k}}{k !} \frac{\Gamma(k+1)}{\Gamma(1+\beta k) \Gamma\left(-\frac{\alpha}{2} k\right)}\left(\tau^{\frac{\alpha}{2}}\right)^{-k-\frac{n}{\alpha}} .
$$

We thus have obtained a representation of $f_{2}$ in terms of the four-parameter Wright function (Equation (30)):

$$
f_{2}(\tau)=\frac{\alpha}{2} \tau^{-n / 2} W_{(1, \beta),(0,-\alpha / 2)}\left(-\tau^{-\alpha / 2}\right),
$$

which is valid under the condition $\beta>\alpha / 2$.

Now we take into consideration the Mellin-Barnes integral given by Equation (38), Equations (40) and (43), and the Mellin transform convolution theorem, and thus we obtain the integral representation given by Equation (36).

The same procedure can be applied for other representations of the kernel function $L_{\alpha, \beta, n}(s)$ as a product of two factors. We again start with the Mellin-Barnes integral given by Equation (25) and perform the variables' substitution $s \rightarrow \alpha s$. Then we obtain the representation

$$
G_{\alpha, \beta, n}(\mathrm{x}, t)=\frac{t^{-\frac{\beta n}{\alpha}}}{(4 \pi)^{\frac{n}{2}}} \frac{1}{2 \pi i} \int_{\gamma-i \infty}^{\gamma+i \infty} \frac{\Gamma\left(\frac{\alpha}{2} s\right) \Gamma\left(\frac{n}{\alpha}-s\right) \Gamma\left(1-\frac{n}{\alpha}+s\right)}{\Gamma\left(1-\frac{\beta}{\alpha} n+\beta s\right) \Gamma\left(\frac{n}{2}-\frac{\alpha}{2} s\right)}\left(z^{\alpha}\right)^{-s} d s, z=\frac{|x|}{2 t^{\frac{\beta}{\alpha}}} .
$$


The next step is a representation of the kernel function of the previous integral as a product of two factors:

$$
L_{\alpha, \beta, n}(s)=\frac{\Gamma\left(1-\frac{n}{\alpha}+s\right)}{\Gamma\left(\frac{n}{2}-\frac{\alpha}{2} s\right)} \times \frac{\Gamma\left(\frac{\alpha}{2} s\right) \Gamma\left(\frac{n}{\alpha}-s\right)}{\Gamma\left(1-\frac{\beta}{\alpha} n+\beta s\right)} .
$$

Now we calculate the inverse Mellin integral transforms of the factors. For the first factor, we employ the same technique as above and obtain the series representation

$$
f_{1}(\tau)=\frac{1}{2 \pi i} \int_{\gamma-i \infty}^{\gamma+i \infty} \frac{\Gamma\left(1-\frac{n}{\alpha}+s\right)}{\Gamma\left(\frac{n}{2}-\frac{\alpha}{2} s\right)} \tau^{-s} d s=\sum_{k=0}^{\infty} \frac{(-1)^{k}}{k !} \frac{1}{\Gamma\left(\frac{\alpha}{2}+\frac{\alpha}{2} k\right)} \tau^{k+1-\frac{n}{\alpha}}
$$

Thus the function $f_{1}$ can be represented in terms of the Wright function (Equation (27)):

$$
f_{1}(\tau)=\tau^{1-\frac{n}{\alpha}} W_{\frac{\alpha}{2}, \frac{\alpha}{2}}(-\tau)
$$

As for the second factor, we first obtain the series representation:

$$
f_{2}(\tau)=\frac{1}{2 \pi i} \int_{\gamma-i \infty}^{\gamma+i \infty} \frac{\Gamma\left(\frac{\alpha}{2} s\right) \Gamma\left(\frac{n}{\alpha}-s\right)}{\Gamma\left(1-\frac{\beta}{\alpha} n+\beta s\right)} d s=\sum_{k=0}^{\infty} \frac{(-1)^{k}}{k !} \frac{\Gamma\left(\frac{n}{2}+\frac{\alpha}{2} k\right)}{\Gamma(1+\beta k)} \tau^{-k-\frac{n}{\alpha}}
$$

and then its representation in terms of the generalized Wright function (Equation (32)):

$$
f_{2}(\tau)=\tau^{-\frac{n}{\alpha}}{ }_{1} \Psi_{1}\left[\begin{array}{c}
\left(\frac{n}{2}, \frac{\alpha}{2}\right) \\
(1, \beta)
\end{array} ;-\frac{1}{\tau}\right] .
$$

Combining Equations (44), (47), and (49) together and applying the Mellin convolution theorem, we finally arrive at the integral representation given by Equation (37) of the fundamental solution in terms of the Wright function and the generalized Wright function.

\section{New Closed-Form Formulas for Particular Cases of the Fundamental Solution}

In the paper [9], the Mellin-Barnes representations of the fundamental solution to the multi-dimensional time- and space-fractional diffusion-wave equation were employed to derive some new particular cases of the solution in terms of the elementary functions and the special functions of the Wright type. In particular, the closed-form formulas for the fundamental solution to the neutral-fractional diffusion equation $(\beta=\alpha$ in Equation (1)) in terms of elementary functions were deduced for the odd-dimensional case $(n=1,3, \ldots)$. In this section, we derive among other things a representation of the fundamental solution to the neutral-fractional diffusion equation in the two-dimensional case in terms of the four-parameter Wright function (Equation (30)).

Theorem 2. The first fundamental solution to the multi-dimensional space- and time-fractional diffusion Equation (1) can be represented in terms of the Wright-type functions:

(a) For $\beta=\alpha$ and $n=2$ under the condition $1<\alpha \leq 2$ :

$$
G_{\alpha, \alpha, 2}(x, t)= \begin{cases}\frac{|x|^{\alpha-2}}{\sqrt{\pi} t^{\alpha}} W_{\left(\frac{1}{2}-\frac{\alpha}{2},-\frac{\alpha}{2}\right),\left(\frac{\alpha}{2}, \frac{\alpha}{2}\right)}\left(-\left(\frac{|x|}{t}\right)^{\alpha}\right) & \text { if }|x|<t, \\ \frac{|x|^{-2}}{\sqrt{\pi}} W_{\left(0,-\frac{\alpha}{2}\right),\left(\frac{1}{2}, \frac{\alpha}{2}\right)}\left(-\left(\frac{t}{|x|}\right)^{\alpha}\right) & \text { if }|x|>t .\end{cases}
$$

(b) For $\beta=\frac{3}{2} \alpha$ and $n=2$ under the condition $1<\alpha \leq \frac{4}{3}$ :

$$
G_{\alpha, \frac{3}{2} \alpha, 2}(x, t)=\frac{\sqrt{3}}{2 \pi^{2}|x|^{2}}{ }_{1} \Psi_{3}\left[\begin{array}{c}
(1,1) \\
\left(\frac{1}{3}, \frac{\alpha}{2}\right),\left(\frac{2}{3}, \frac{\alpha}{2}\right),\left(0,-\frac{\alpha}{2}\right)
\end{array} ;-\left(\frac{|x|}{2(3 t)^{\frac{3}{2}}}\right)^{\alpha}\right] .
$$


Proof. Once again we start with the Mellin-Barnes integral representation given by Equation (25), which for $\beta=\alpha$ and $n=2$, takes the following form:

$$
G_{\alpha, \alpha, 2}(\mathrm{x}, t)=\frac{1}{\alpha} \frac{t^{-2}}{4 \pi} \frac{1}{2 \pi i} \int_{\gamma-i \infty}^{\gamma+i \infty} \frac{\Gamma\left(\frac{s}{2}\right) \Gamma\left(\frac{2}{\alpha}-\frac{s}{\alpha}\right) \Gamma\left(1-\frac{2}{\alpha}+\frac{s}{\alpha}\right)}{\Gamma(-1+s) \Gamma\left(1-\frac{s}{2}\right)}\left(\frac{|\mathrm{x}|}{2 t}\right)^{-s} d s
$$

The general theory of Mellin-Barnes integrals (see, e.g., [22]) states that for $|x| \leq 2 t$, a series representation of Equation (52) can be obtained by transforming the contour of integration in the integral on the right-hand side of Equation (52) to the loop $L_{-\infty}$ starting and ending at $-\infty$ and encircling all poles of the functions $\Gamma\left(\frac{s}{2}\right)$ and $\Gamma\left(1-\frac{2}{\alpha}+\frac{s}{\alpha}\right)$. The problem now is that we have to take into consideration the cases in which some of the poles of $\Gamma\left(\frac{s}{2}\right)$ coincide with the poles $\Gamma\left(1-\frac{2}{\alpha}+\frac{s}{\alpha}\right)$, making the series representation become very complicated.

To avoid this problem, we aim to "eliminate" one of these gamma functions. The application of the duplication formula for the gamma function:

$$
\Gamma(2 s)=\frac{2^{2 s-1}}{\sqrt{\pi}} \Gamma(s) \Gamma\left(s+\frac{1}{2}\right)
$$

to the function $\Gamma(-1+s)$ (one of the Gamma-functions in the denominator of the kernel function from the integral in Equation (52)) results in the following representation:

$$
\Gamma(1-s)=\Gamma\left(2\left(-\frac{1}{2}+\frac{s}{2}\right)\right)=\frac{2^{s-2}}{\sqrt{\pi}} \Gamma\left(-\frac{1}{2}+\frac{s}{2}\right) \Gamma\left(\frac{s}{2}\right) .
$$

Now we substitute the previous formula into the integral in Equation (52) and obtain another Mellin-Barnes representation:

$$
G_{\alpha, \alpha, 2}(x, t)=\frac{1}{\alpha} \frac{t^{-2}}{\sqrt{\pi}} \frac{1}{2 \pi i} \int_{\gamma-i \infty}^{\gamma+i \infty} \frac{\Gamma\left(\frac{2}{\alpha}-\frac{s}{\alpha}\right) \Gamma\left(1-\frac{2}{\alpha}+\frac{s}{\alpha}\right)}{\Gamma\left(-\frac{1}{2}+\frac{s}{2}\right) \Gamma\left(1-\frac{s}{2}\right)}\left(\frac{|x|}{t}\right)^{-s} d s .
$$

In contrast to the representation given by Equation (52), the numerator of the kernel function in Equation (53) has just one gamma function with the poles tending to $-\infty$ and one gamma function with the poles tending to $+\infty$, and thus this representation is very suitable for the derivation of a series representation of $G_{\alpha, \alpha, 2}$.

To proceed, the variables' substitution $s \rightarrow \alpha s$ is first employed in the integral from Equation (53). We then obtain the representation

$$
G_{\alpha, \alpha, 2}(\mathrm{x}, t)=\frac{t^{-2}}{\sqrt{\pi}} \frac{1}{2 \pi i} \int_{\gamma-i \infty}^{\gamma+i \infty} \frac{\Gamma\left(\frac{2}{\alpha}-s\right) \Gamma\left(1-\frac{2}{\alpha}+s\right)}{\Gamma\left(-\frac{1}{2}+\frac{\alpha}{2} s\right) \Gamma\left(1-\frac{\alpha}{2} s\right)}\left(\left(\frac{|\mathrm{x}|}{t}\right)^{\alpha}\right)^{-s} d s .
$$

To obtain the series representation of the Mellin-Barnes integral (Equation (54)), we have to consider two cases:

(i) $|x|<t$;

(ii) $|\mathrm{x}|>t$.

In the first case, the contour of integration in the integral on the right-hand side of Equation (54) can be transformed to the loop $L_{-\infty}$ starting and ending at $-\infty$ and encircling all poles of the function 
$\Gamma\left(1-\frac{2}{\alpha}+s\right)$. Taking into account the Jordan lemma and the formula for the residuals of the gamma function, the Cauchy residue theorem leads to the following series representation of $G_{\alpha, \alpha, 2}$ :

$$
G_{\alpha, \alpha, 2}(\mathrm{x}, t)=\frac{t^{-2}}{\sqrt{\pi}} \sum_{k=0}^{\infty} \frac{(-1)^{k}}{k !} \frac{k !\left(\left(\frac{|\mathrm{x}|}{t}\right)^{\alpha}\right)^{1+k-\frac{2}{\alpha}}}{\Gamma\left(\frac{1}{2}-\frac{\alpha}{2}-\frac{\alpha}{2} k\right) \Gamma\left(\frac{\alpha}{2}+\frac{\alpha}{2} k\right)} .
$$

We thus arrive at the closed-form formula:

$$
G_{\alpha, \alpha, 2}(\mathrm{x}, t)=\frac{|\mathrm{x}|^{\alpha-2}}{\sqrt{\pi} t^{\alpha}} W_{\left(\frac{1}{2}-\frac{\alpha}{2},-\frac{\alpha}{2}\right),\left(\frac{\alpha}{2}, \frac{\alpha}{2}\right)}\left(-\left(\frac{|\mathrm{x}|}{t}\right)^{\alpha}\right)
$$

in terms of the four-parameter Wright function (Equation (30)) that is valid for $|\mathrm{x}|<t$.

In the case $|x|>t$, the contour of integration in the integral on the right-hand side of Equation (54) can be transformed to the loop $L_{+\infty}$ starting and ending at $+\infty$ and encircling all poles of the function $\Gamma\left(\frac{2}{\alpha}-s\right)$. Proceeding as in case i), we first obtain a series representation of $G_{\alpha, \alpha, 2}$ in the form

$$
G_{\alpha, \alpha, 2}(\mathrm{x}, t)=\frac{t^{-2}}{\sqrt{\pi}} \sum_{k=0}^{\infty} \frac{(-1)^{k}}{k !} \frac{k !\left(\left(\frac{|\mathrm{x}|}{t}\right)^{\alpha}\right)^{-k-\frac{2}{\alpha}}}{\Gamma\left(-\frac{\alpha}{2} k\right) \Gamma\left(\frac{1}{2}+\frac{\alpha}{2} k\right)}
$$

and then obtain the closed-form formula:

$$
G_{\alpha, \alpha, 2}(x, t)=\frac{|x|^{-2}}{\sqrt{\pi}} W_{\left(0,-\frac{\alpha}{2}\right),\left(\frac{1}{2}, \frac{\alpha}{2}\right)}\left(-\left(\frac{t}{|x|}\right)^{\alpha}\right)
$$

in terms of the four-parameter Wright function that is valid for $|x|>t$.

Combining Equations (56) and (58), the obtain the representation given by Equation (50) of the fundamental solution $G_{\alpha, \alpha, 2}$ in terms of the four-parameter Wright function.

In the case $|\mathbf{x}|=t$, both series are divergent, and the problem of determining a series representation of $G_{\alpha, \alpha, 2}$ is more complicated; it will be considered elsewhere.

The method described above can be used for the derivation of other closed-form formulas for particular cases of the fundamental solution $G_{\alpha, \beta, n}$ in terms of the Wright-type functions. For example, we consider the case $\beta=\frac{3}{2} \alpha$ and $n=2$ (because of the condition $\beta \leq 2$, in this case, the inequalities $1<\alpha \leq \frac{4}{3}$ have to be satisfied). The Mellin-Barnes representation of $G_{\alpha, \frac{3}{2} \alpha, 2}$ is as follows:

$$
G_{\alpha, \frac{3}{2} \alpha, 2}(\mathrm{x}, t)=\frac{1}{\alpha} \frac{t^{-3}}{4 \pi} \frac{1}{2 \pi i} \int_{\gamma-i \infty}^{\gamma+i \infty} \frac{\Gamma\left(\frac{s}{2}\right) \Gamma\left(\frac{2}{\alpha}-\frac{s}{\alpha}\right) \Gamma\left(1-\frac{2}{\alpha}+\frac{s}{\alpha}\right)}{\Gamma\left(-2+\frac{3}{2} s\right) \Gamma\left(1-\frac{s}{2}\right)}\left(\frac{|\mathbf{x}|}{2 t^{\frac{3}{2}}}\right)^{-s} d s .
$$

To proceed, we apply the multiplication formula for the gamma function:

$$
\Gamma(m s)=m^{m s-\frac{1}{2}}(2 \pi)^{\frac{1-m}{2}} \prod_{k=0}^{m-1} \Gamma\left(s+\frac{k}{m}\right), m=2,3,4, \ldots
$$

with $m=3$ to the gamma function $\Gamma\left(-2+\frac{3}{2} s\right)$ from the denominator of the kernel function from the Mellin-Barnes representation given by Equation (59). We thus obtain the representation

$$
\Gamma\left(-2+\frac{3}{2} s\right)=\Gamma\left(3\left(-\frac{2}{3}+\frac{1}{2} s\right)\right)=3^{-\frac{5}{2}+\frac{3}{2} s}(2 \pi)^{-1} \Gamma\left(-\frac{2}{3}+\frac{1}{2} s\right) \Gamma\left(-\frac{1}{3}+\frac{1}{2} s\right) \Gamma\left(\frac{1}{2} s\right) .
$$


By applying this formula to Equation (59) and by the variables' substitution $s \rightarrow \alpha s$, we arrive at the following Mellin-Barnes representation:

$$
G_{\alpha, \frac{3}{2} \alpha, 2}(\mathrm{x}, t)=\frac{t^{-3}}{4 \pi} \frac{3^{-\frac{5}{2}}}{2 \pi} \frac{1}{2 \pi i} \int_{\gamma-i \infty}^{\gamma+i \infty} \frac{\Gamma\left(\frac{2}{\alpha}-s\right) \Gamma\left(1-\frac{2}{\alpha}+s\right)}{\Gamma\left(-\frac{2}{3}+\frac{\alpha}{2} s\right) \Gamma\left(-\frac{1}{3}+\frac{\alpha}{2} s\right) \Gamma\left(1-\frac{\alpha}{2} s\right)}\left(\left(\frac{|x|}{2(3 t)^{\frac{3}{2}}}\right)^{\alpha}\right)^{-s} d s
$$

Using the technique presented above, the representation given by Equation (60) leads first to a series representation of $G_{\alpha, \frac{3}{2} \alpha, 2}$ in the following form:

$$
G_{\alpha, \frac{3}{2} \alpha, 2}(\mathrm{x}, t)=\frac{\sqrt{3}}{2 \pi^{2}|\mathrm{x}|^{2}} \sum_{k=0}^{\infty} \frac{\left(-\left(\frac{|\mathrm{x}|}{2(3 t)^{\frac{3}{2}}}\right)^{\alpha}\right)^{k}}{\Gamma\left(\frac{1}{3}+\frac{\alpha}{2} k\right) \Gamma\left(\frac{2}{3}+\frac{\alpha}{2} k\right) \Gamma\left(-\frac{\alpha}{2} k\right)},
$$

which can be represented as a particular case of the generalized Wright function (Equation (51)).

\section{Discussion}

This paper is devoted to some applications of the Mellin-Barnes integral representations of the fundamental solution to the multi-dimensional space- and time-fractional diffusion-wave equation for the analysis of its properties. In particular, this representation is used to obtain two new representations of the fundamental solution in the form of the Mellin convolution of the special functions of Wright type and for the derivation of some new closed-form formulas for particular cases of the fundamental solution. Among other things, the open problem of the representation of the fundamental solution to the two-dimensional neutral-fractional diffusion-wave equation in terms of the known special functions is solved. The potential of the Mellin-Barnes integral representation of the fundamental solution to the multi-dimensional space- and time-fractional diffusion-wave equation is of course not yet ladled. It can be used among other things for the derivation of the new closed-form formulas for its particular cases, for asymptotic formulas for the fundamental solution, and for relationships between the fundamental solutions for different values of the derivative orders $\alpha$ and $\beta$. These problems will be considered elsewhere in further publications.

Conflicts of Interest: The author declares no conflict of interest.

\section{References}

1. Kochubei, A.N. Fractional-order diffusion. Differ. Equ. 1990, 26, 485-492.

2. Schneider, W.R.; Wyss, W. Fractional diffusion and wave equations. J. Math. Phys. 1989, 30, 134-144.

3. Hanyga, A. Multidimensional solutions of space-fractional diffusion equations. Proc. R. Soc. Lond. A 2001, 457, 2993-3005.

4. Hanyga, A. Multi-dimensional solutions of space-time-fractional diffusion equations. Proc. R. Soc. Lond. A 2002, 458, 429-450.

5. Hanyga, A. Multidimensional solutions of time-fractional diffusion-wave equations. Proc. R. Soc. Lond. A 2002, 458, 933-957.

6. Luchko, Y. Fractional wave equation and damped waves. J. Math. Phys. 2013, 54, 031505.

7. Luchko, Y. Multi-dimensional fractional wave equation and some properties of its fundamental solution. Commun. Appl. Ind. Math. 2014, 6, e485.

8. Luchko, Y. Wave-diffusion dualism of the neutral-fractional processes. J. Comput. Phys. 2015, 293, 40-52.

9. Boyadjiev, L.; Luchko, Y. Mellin integral transform approach to analyze the multidimensional diffusion-wave equations. Chaos Solitons Fractals 2017, 102, 127-134.

10. Boyadjiev, L.; Luchko, Y. Multi-dimensional $\alpha$-fractional diffusion-wave equation and some properties of its fundamental solution. Comput. Math. Appl. 2017, 73, 2561-2572. 
11. Mainardi, F.; Luchko, Y.; Pagnini, G. The fundamental solution of the space-time fractional diffusion equation. Fract. Calc. Appl. Anal. 2001, 4, 153-192.

12. Saichev, A.; Zaslavsky, G. Fractional kinetic equations: Solutions and applications. Chaos 1997, 7, 753-764.

13. Samko, S.G.; Kilbas, A.A.; Marichev, O.I. Fractional Integrals and Derivatives: Theory and Applications; Gordon and Breach: New York, NY, USA, 1993.

14. Ferreira, M.; Vieira, N. Fundamental solutions of the time fractional diffusion-wave and parabolic Dirac operators. J. Math. Anal. Appl. 2016, 447, 329-353.

15. Kochubei, A.N. Cauchy problem for fractional diffusion-wave equations with variable coefficients. Appl. Anal. 2014, 93, 2211-2242.

16. Luchko, Y. Some uniqueness and existence results for the initial-boundary-value problems for the generalized time-fractional diffusion equation. Comput. Math. Appl. 2010, 59, 1766-1772.

17. Eidelman, S.D.; Kochubei, A.N. Cauchy problem for fractional diffusion equations. J. Differ. Equ. 2004, 199, 211-255.

18. Luchko, Y. Operational method in fractional calculus. Fract. Calc. Appl. Anal. 1999, 2, 463-489.

19. Erdélyi, A. Higher Transcendental Functions, Volume 3; McGraw-Hill: New York, NY, USA, 1955.

20. Erdélyi, A. Higher Transcendental Functions, Volume 2; McGraw-Hill: New York, NY, USA, 1953.

21. Luchko, Y.; Kiryakova, V. The Mellin integral transform in fractional calculus. Fract. Calc. Appl. Anal. 2013, $16,405-430$.

22. Marichev, O.I. Handbook of Integral Transforms of Higher Transcendental Functions, Theory and Algorithmic Tables; Ellis Horwood: Chichester, UK, 1983.

23. Fox, C. The G- and H-functions as symmetrical Fourier kernels. Trans. Am. Math. Soc. 1961, 98, 395-429.

24. Kilbas, A.A.; Saigo, M. H-Transform. Theory and Applications; Chapman and Hall: Boca Raton, FL, USA, 2004.

25. Kiryakova, V. Generalized Fractional Calculus and Applications; Longman: Harlow, UK, 1994.

26. Mainardi, F.; Pagnini, G. Salvatore Pincherle: The pioneer of the Mellin-Barnes integrals. J. Comput. Appl. Math. 2003, 153, 331-342.

27. Mathai, A.M.; Saxena, R.K. The H-Functions with Applications in Statistics and Other Disciplines; John Wiley: New York, NY, USA, 1978.

28. Yakubovich, S.; Luchko, Y. The Hypergeometric Approach to Integral Transforms and Convolutions; Kluwer Academic Publishers: Dordrecht, The Netherlands, 1994.

29. Mainardi, F. Fractional relaxation-oscillation and fractional diffusion-wave phenomena. Chaos Solitons Fractals 1996, 7, 1461-1477.

30. Gorenflo, R.; Luchko, Y.; Mainardi, F. Analytical properties and applications of the Wright function. Fract. Calc. Appl. Anal. 1999, 2, 383-414.

31. Gorenflo, R.; Luchko, Y.; Mainardi, F. Wright functions as scale-invariant solutions of the diffusion-wave equation. J. Comput. Appl. Math. 2000, 118, 175-191.

32. Luchko, Y. Algorithms for evaluation of the Wright function for the real arguments' values. Fract. Calc. Appl. Anal. 2008, 11, 57-75.

33. Luchko, Y.; Mainardi, F. Cauchy and signaling problems for the time-fractional diffusion-wave equation. ASME J. Vib. Acoust. 2014, 135, doi:10.1115/1.4026892.

34. Mainardi, F. Fractional Calculus and Waves in Linear Viscoelasticity; Imperial College Press: London, UK, 2010.

35. Pagnini, G. The M-Wright function as a generalization of the Gaussian density for fractional diffusion processes. Fract. Calc. Appl. Anal. 2013, 16, 436-453.

36. Stanković, B. On the function of E.M. Wright. Publ. l'Inst. Math. Beogr. Nouv. Sèr. 1970, 10, 113-124.

37. Wright, E.M. On the coefficients of power series having exponential singularities. J. Lond. Math. Soc. 1933, 8 , 71-79.

38. Wright, E.M. The asymptotic expansion of the generalized Bessel function. Proc. Lond. Math. Soc. 1935, 38, 257-270.

39. Gorenflo, R.; Mainardi, F.; Srivastava, H.M. Special functions in fractional relaxation-oscillation and fractional diffusion-wave phenomena. In Proceedings VIII International Colloquium on Differential Equations; Bainov, D., Ed.; VSP: Utrecht, The Netherlands, 1998; pp. 195-202.

40. Wright, E.M. The generalized Bessel function of order greater than one. Quart. J. Math. 1940, 11, 36-48. 
41. Mainardi, F. Fractional calculus: some basic problems in continuum and statistical mechanics. In Fractals and Fractional Calculus in Continuum Mechanics; Carpinteri, A., Mainardi, F., Eds.; Springer: Wien, Austria, 1997; pp. 291-348.

42. Mainardi, F.; Tomirotti, M. On a special function arising in the time fractional diffusion-wave equation. In Transform Methods and Special Functions; Rusev, P., Dimovski, I., Kiryakova, V., Eds; Science Culture Technology: Singapore, 1995; pp. 171-183.

43. Wright, E.M. The asymptotic expansion of the generalized hypergeometric function. J. Lond. Math. Soc. 1935, 10, 287-293.

44. Luchko, Y.; Gorenflo, R. Scale-invariant solutions of a partial differential equation of fractional order. Fract. Calc. Appl. Anal. 1998, 1, 63-78.

45. Gorenflo, R.; Kilbas, A.A.; Mainardi, F.; Rogosin, S.V. Mittag-Leffler Functions, Related Topics and Applications; Springer: Berlin, Germany, 2014.

(C) 2017 by the author. Licensee MDPI, Basel, Switzerland. This article is an open access article distributed under the terms and conditions of the Creative Commons Attribution (CC BY) license (http:/ / creativecommons.org/licenses/by/4.0/). 